\title{
Psychological distress as a risk factor for death from cerebrovascular disease
}

\author{
Mark Hamer PhD, Mika Kivimaki PhD, Emmanuel Stamatakis PhD, G. David Batty PhD
}

See related commentary by Pan and Rexrode on page 1453 and at www.cmaj.ca/lookup/doi/10.1503/cmaj.121288

\begin{abstract}
- Abstract
Background: Little is known about psychological risk factors in cerebrovascular disease. We examined the association between psychological distress and risk of death due to cerebrovascular disease.

Methods: We obtained data from 68652 adult participants of the Health Survey for England (mean age 54.9 [standard deviation 13.9] yr, $45.0 \%$ male sex) with no known history of cardiovascular diseases at baseline. We used the 12-item General Health Questionnaire (GHQ-12) to assess the presence of psychological distress. We followed participants for eight years for cause-specific death using linkage to national registers.

Results: There were 2367 deaths due to cardiovascular disease during follow-up. Relative to participants with no symptoms of psychological distress (GHQ-12 score 0) at baseline,

people with psychological distress (GHQ-12 score $\geq 4,14.7 \%$ of participants) had an increased risk of death from cerebrovascular disease (adjusted hazard ratio [HR] 1.66, 95\% confidence interval [CI] 1.32-2.08) and ischemic heart disease (adjusted HR 1.59, $95 \% \mathrm{Cl} 1.34-1.88)$. There was also evidence of a dose-response effect with increasing GHQ12 score ( $p$ for trend $<0.001$ in all analyses). Associations were only marginally attenuated after we adjusted for possible confounders, including socioeconomic status, smoking and use of antihypertensive medications.

Interpretation: Psychological distress was associated with increased risk of death due to cerebrovascular disease in a large populationrepresentative cohort. These data suggest that the cardiovascular effects of psychological distress are not limited to coronary artery disease.
\end{abstract}

Competing interests: None declared.

This article has been peer reviewed.

Correspondence to:

Mark Hamer,

m.hamer@ucl.ac.uk

CMAJ 2012. DOI:10.1503 /cmaj.111719
$\mathrm{P}$ sychological constructs are becoming increasingly recognized as risk factors and triggers for cardiovascular events. ${ }^{1}$ To date, most cardiovascular research has focused on coronary artery disease rather than cerebrovascular disease. Several studies have examined the association between symptoms of depression and risk of stroke, ${ }^{2-11}$ although the results have been inconsistent, and effect sizes are generally modest. ${ }^{12}$ For example, some evidence from epidemiological studies has shown a relation between depression and incident stroke..$^{2-7}$ However, a recent study showed an association between depression and coronary artery disease but not cerebrovascular disease. ${ }^{11}$ This finding is consistent with other analyses that have also found null associations for stroke outcomes. ${ }^{8-10}$ Other investigators have suggested that psychological distress is a more robust predictor of stroke than are symptoms of depression. ${ }^{13}$

In addition to a paucity of evidence linking psychological distress with risk of stroke, extant evidence is hampered by a series of methodolog- ical problems. These limitations include small sample sizes (including rarity of outcome), a limited range of confounding data, an unknown influence of existing comorbidity on psychological distress (reverse causality) and few data from women. We addressed these shortcomings by examining the association between psychological distress and risk of death due to cerebrovascular disease in a large, well-characterized cohort of men and women representative of the general population.

\section{Methods}

\section{Participants and study design}

We drew our participants from those recruited for the Health Survey for England, a representative, general population-based study sampling people living in households. ${ }^{14}$ Participants are selected using a multistage stratified probability design to give a representative sample of the target population in each survey year. Stratification is based on geography rather than individual characteristics, 
with postal codes selected at the first stage and household addresses selected at the second stage. The overall rate of response ranged between $60 \%$ and $90 \%$ in different survey years.

We used a prospective cohort design. We pooled participants from 10 different survey years (1994-2004; Appendix 1, available at www .cmaj.ca/lookup/suppl/doi:10.1503/cmaj.111719/-/ DC1). We linked study participants to mortality data from the National Health Service and followed them until Feb. 28, 2008. Study participants gave their full informed consent. The study was approved by the London Multicentre Research Ethics Committee.

\section{Primary outcome measure}

Our primary outcome measure was death due to cerebrovascular disease. Our primary exposure variable of interest was psychological distress.

During a household visit, interviewers collected information using computer-assisted personal interviewing modules. Psychological distress was measured using the 12-item version of the General Health Questionnaire (GHQ-12), a widely used measure of psychological distress in population studies. ${ }^{15}$ The GHQ-12 is a unidimensional scale ${ }^{16}$ consisting of items relating to anxiety, depression, social dysfunction and loss of confidence. Answers are interpreted according to a 4-point response scale scored using a bimodal method (symptom present "not at all" or "same as usual $=0$; "more than usual" or "much more than usual" $=1$ ).

To determine whether there was a doseresponse relation, we categorized the results from the GHQ-12 into three groups $(0,1-3$ and 4 points); a score of 4 or more denoted a case. ${ }^{15}$ This definition has been validated against standardized psychiatric interviews and has been strongly associated with various psychological disorders, such as depression and anxiety. ${ }^{17}$

\section{Other covariables}

Interviewers collected information on selfreported smoking status (current, former or never), alcohol consumption (units per week), moderate to vigorous physical activity (frequency per week), ethnicity (white, black, South Asian and other) and socioeconomic status as indexed by occupational social class (professional/intermediate, skilled non-manual, skilled manual and part-skilled/unskilled). In addition, interviewers measured participants' height and weight to calculate body mass index (BMI). During a separate visit, nurses collected data on history of clinician-diagnosed cardiovascular disease (stroke, angina or heart attack), diabetes and the use of antihypertensive medications.

\section{Determining cause of death}

We classified underlying causes of death using information collected from participants' death certificates together with any additional information provided by the certifying physicians (e.g., secondary cause of death). We recorded diagnoses for primary cause of death using the International Classification of Diseases, ninth (ICD9) and tenth (ICD-10) revisions (ischemic heart disease: ICD-9 codes 410-414, ICD-10 codes I20-I25; cerebrovascular disease: ICD-9 codes 430-438, ICD-10 codes I60-I69).

\section{Statistical analysis}

We used $\chi^{2}$ tests and analyses of variance to determine differences in baseline characteristics across the three psychological distress groups. We used Cox proportional hazards models to compute hazard ratios (HRs) with accompanying 95\% confidence intervals (CIs) for the association between death from cardiovascular disease and psychological distress. We used age as the time scale, with entry delayed to age at cohort inception; for participants with no record of an event (i.e., those who did not die from cardiovascular disease or from some other cause), the data were censored at Feb. 28, 2008. We examined the proportional hazards assumption by calculating time-dependent interaction terms between GHQ12 score and the logarithm of the follow-up period, which were all nonsignificant. With no clear differences in our results between men and women, we pooled the data and adjusted for sex, age, ethnicity, socioeconomic status, smoking status and use of antihypertensive medications. The rationale for the inclusion of these covariables was that they were both important predictors of cardiovascular disease and associated with psychological distress. We further adjusted for survey year to address any concerns about time lag effects. Given that there were considerable amounts of missing data on alcohol consumption, physical activity, physician-diagnosed diabetes and BMI, we performed additional sensitivity analyses on a subsample in which these covariables were added to the model.

\section{Results}

The initial study sample included 96605 adults, $10065(10.4 \%)$ of whom did not consent to mortality follow-up and were removed from the analyses. Nonconsenting adults were older than those who gave consent (64.3 v. $56.1 \mathrm{yr}, p<0.001)$.

Further exclusion criteria included cliniciandiagnosed cardiovascular disease at baseline $(n=$ 4160), and missing data on the exposure variable (i.e., psychological distress, as measured by the 
GHQ-12) ( $n=13728)$. The final cohort comprised 68652 adults (mean age 54.9 [standard deviation 13.9] yr, $45.0 \%$ male sex, $96.1 \%$ white ethnicity). Participants who gave their consent but who were excluded from the analysis were older (60.7 v. $54.7 \mathrm{yr})$ and more likely to be men $(46.5 \% \mathrm{v}$. $44.6 \%$ ) than those included in the final cohort.

Psychological distress was evident in $14.7 \%$ of participants. People reporting psychological distress were younger and were more likely to be women, come from lower/working classes, smoke and use antihypertensive medications (Table 1) than participants not reporting such distress.

Over an average of 8.1 years' follow-up (maximum $14 \mathrm{yr}$ ), there were 2367 deaths due to cardiovascular disease (1010 deaths from ischemic heart disease, 562 deaths from cerebrovascular disease, 795 other cardiovascular-related deaths). Psychological distress was associated with death from cardiovascular disease, and the relation remained consistent for specific disease outcomes, including ischemic heart disease and cerebrovascular disease (Table 2). Other independent risk factors for death due to cerebrovascular disease included age (per-year increase: HR $=1.16$, 95\% CI 1.15-1.17), smoking (multivariable $\mathrm{HR}=1.71,95 \%$ CI $1.33-2.19)$ and use of antihypertensive medications (multivariable $\mathrm{HR}=$ $1.26,95 \%$ CI 1.04-1.52). The association between psychological distress and cerebrovascular disease was only marginally attenuated after adjusting for possible confounders (Table 2).

\section{Sensitivity analysis}

We wanted to examine the potential role of blood pressure as a mechanism in explaining the link between psychological distress and cerebrovascular disease. Using data from the subsample of participants for whom information on blood pressure was available $(n=52481,432$ of whom died from cerebrovascular disease), we found that the association between psychological distress and cerebrovascular disease was similar to that of the main cohort (age- and sex-adjusted HR 1.64, 95\% CI 1.27-2.12); however, adjusting for systolic blood pressure did not attenuate the effect estimate (HR 1.69, 95\% CI 1.30-2.18).

We performed a second analysis using data from the subsample of participants for whom data on covariables including alcohol consumption, physical activity, physician-diagnosed diabetes and BMI $(n=21385)$ were available, but additional adjustments for these variables only marginally changed the results (Appendix 2, available at www.cmaj.ca/lookup/suppl/doi:10 .1503/cmaj.111719/-/DC1.).

We performed a further sensitivity analysis in which missing data were estimated using multi- ple imputation methods. The missing values for covariables were imputed using five imputations based on maximum-likelihood estimates. We saw minimal differences between the original results and those found using the imputed dataset. For example, for an increase of one standard deviation in GHQ-12 score, the risk of death due to cerebrovascular disease was 1.09 (95\% CI 1.04-1.15) using the original data and 1.12 (85\% CI 1.08-1.17) using the imputed data.

Our final sensitivity analysis involved removing all cardiovascular disease deaths in the first two years $(n=411)$ after the inception of each individual survey, which did not appreciably change any of our results.

\section{Interpretation}

We saw an association between psychological distress and risk of cerebrovascular disease among our participants, all of whom had been free from cardiovascular disease at baseline. This association was similar in size to the association between psychological distress and ischemic heart disease in the same group. Our results were largely unchanged by controlling for a range of covariables relevant to cardiovascular disease outcomes.

To date, most of the research in this area has focused on coronary artery disease. Previous studies have been limited by the rarity of cerebrovascular events, a limited range of covariables, the unknown influence of existing comorbidity on psychological distress (reverse causality) and

Table 1: Characteristics of the study participants at baseline $(n=68652)$

\begin{tabular}{|c|c|c|c|}
\hline \multirow[b]{2}{*}{ Characteristic } & \multicolumn{3}{|c|}{ GHQ-12 score } \\
\hline & $\begin{array}{c}0 \\
(n=41665)\end{array}$ & $\begin{array}{c}1-3 \\
(n=16915)\end{array}$ & $\begin{array}{c}\geq 4 \\
(n=10072)\end{array}$ \\
\hline Age, yr, mean (SD) & $55.1(13.5)$ & $55.1(14.4)$ & $53.8(14.1)$ \\
\hline Male, \% & 47.9 & 42.5 & 37.5 \\
\hline \multicolumn{4}{|l|}{ Socioeconomic group, \% } \\
\hline Professional & 40.3 & 38.8 & 34.1 \\
\hline Intermediate & 40.8 & 40.7 & 40.9 \\
\hline Partly skilled/unskilled & 18.9 & 20.5 & 25.0 \\
\hline \multicolumn{4}{|l|}{ Smoking status, $\%$} \\
\hline Never & 42.4 & 40.9 & 39.1 \\
\hline Former & 36.7 & 35.6 & 31.9 \\
\hline Current & 21.0 & 23.5 & 29.0 \\
\hline $\begin{array}{l}\text { Use of antihypertensive } \\
\text { agents, \%* }\end{array}$ & 10.9 & 12.5 & 12.1 \\
\hline White ethnicity, \% & 96.6 & 95.9 & 95.2 \\
\hline \multicolumn{4}{|c|}{$\begin{array}{l}\text { Note: GHQ-12 = } 12 \text {-item General Health Questionnaire. } \\
\text { *Includes diuretics, angiotensin-converting enzyme inhibitors, } \beta \text {-blockers and calcium-channel } \\
\text { blockers. }\end{array}$} \\
\hline
\end{tabular}


few data from women. Our study adds substantially to the extant literature, because it overcomes some of these weaknesses by employing a large, well-characterized cohort with a sufficient number of cardiovascular disease events.

Our results are consistent with what literature does exist. ${ }^{13,18}$ In the European Prospective Investigation into Cancer - Norfolk study, which involved 20627 participants who were strokefree at baseline, psychological distress as measured using the Mental Health Inventory was associated with fatal and nonfatal stroke; depressive symptoms did not show this association. ${ }^{13}$ Although the GHQ-12 includes items covering symptoms of depression, it is generally viewed as a measure of overall mental health; the GHQ12 also includes items covering symptoms of anxiety, social dysfunction, sleep disturbance and loss of confidence. Nevertheless, the effect estimates for psychological distress and cerebrovascular disease we saw are comparable with those seen in a recent meta-analysis for depression and stroke (pooled adjusted HR 1.45,
95\% CI 1.29-1.63). ${ }^{12}$ However, other aspects of psychological dysfunction may have importance. For example, sleeping problems have previously been associated with increased risk of death due to cardiovascular disease, ${ }^{19}$ but the link with anxiety is less clear. ${ }^{20}$

Although the effect estimates of psychological distress were similar for cerebrovascular and ischemic heart disease, the mechanisms might not necessarily be the same because of the differences in pathogenesis of atherosclerotic lesions in coronary and cerebral arteries. ${ }^{21,22}$ Blood pressure is a plausible mechanism linking psychological stress and risk of stroke. For example, men with exaggerated systolic blood pressure reactivity to stress had $72 \%$ greater risk of incident stroke over 11 years follow-up. ${ }^{23}$ Nevertheless, using the same cohort as in the current study, we recently showed that psychological distress is associated with patients knowing that they have hypertension, but not with elevated blood pressure in participants without a diagnosis of hypertension. ${ }^{24}$

Table 2: The association between psychological distress and death due to cardiovascular disease among the study participants $(n=68652)$

\begin{tabular}{|c|c|c|c|}
\hline Mental distress, score on GHQ-12 & Cases, no. & $\begin{array}{l}\text { Age- and sex- } \\
\text { adjusted HR } \\
(95 \% \mathrm{Cl})\end{array}$ & $\begin{array}{l}\text { Multivariable } \\
\text { adjusted* } \mathrm{HR} \\
(95 \% \mathrm{Cl})\end{array}$ \\
\hline \multicolumn{4}{|l|}{ Cerebrovascular disease } \\
\hline 0 & $\begin{array}{c}n=41665 \\
295\end{array}$ & 1.00 & 1.00 \\
\hline $1-3$ & $\begin{array}{c}n=16915 \\
160\end{array}$ & $1.21(1.00-1.47)$ & $1.20(0.99-1.46)$ \\
\hline$\geq 4$ & $\begin{array}{c}n=10072 \\
107\end{array}$ & $1.67(1.34-2.09)$ & $1.66(1.32-2.08)$ \\
\hline$p$ for trend & & $<0.001$ & $<0.001$ \\
\hline \multicolumn{4}{|l|}{ Ischemic heart disease } \\
\hline 0 & $\begin{array}{c}n=41665 \\
548\end{array}$ & 1.00 & 1.00 \\
\hline $1-3$ & $\begin{array}{c}n=16915 \\
288\end{array}$ & $1.31(1.13-1.51)$ & $1.26(1.09-1.46)$ \\
\hline$\geq 4$ & $\begin{array}{c}n=10072 \\
174\end{array}$ & $1.65(1.39-1.96)$ & $1.59(1.34-1.88)$ \\
\hline$p$ for trend & & $<0.001$ & $<0.001$ \\
\hline \multicolumn{4}{|l|}{ All cardiovascular disease } \\
\hline 0 & $\begin{array}{c}n=41665 \\
1273\end{array}$ & 1.00 & 1.00 \\
\hline $1-3$ & $\begin{array}{c}n=16915 \\
652\end{array}$ & $1.21(1.09-1.32)$ & $1.16(1.06-1.28)$ \\
\hline$\geq 4$ & $\begin{array}{c}n=10072 \\
442\end{array}$ & $1.68(1.50-1.87)$ & $1.61(1.44-1.79)$ \\
\hline$p$ for trend & & $<0.001$ & $<0.001$ \\
\hline
\end{tabular}


In addition, adjustment for antihypertensive medications as a proxy of increased blood pressure had minimal effect on the association between distress and cerebrovascular disease in the present analysis. Thus, a possible causal association between distress and hypertension remains doubtful. Other plausible mechanisms might involve inflammatory pathways and dysregulated function of the autonomic nervous system, although existing data relating to psychological distress and cardiovascular disease have been equivocal. ${ }^{25,26}$ There has been a lack of research into the mechanisms that might explain the association between psychological distress and stroke, and further work is needed to address this need.

\section{Limitations}

We found that the size of the association between psychological distress and death from cardiovascular disease diminished as the duration of follow-up increased (Appendix 1), which may suggest reverse causality. That is, undiagnosed somatic illness will be associated with both an increased prevalence of psychological distress and an increased risk of death. The impact of hidden somatic illness will diminish as the length of follow-up increases, because people with such disorders die - potentially resulting in the trend we saw in the current study. In addition, with greater time between measuring psychological distress and death from cardiovascular disease, there is greater opportunity for other risk factors to play a role in the association. An additional explanation for the dilution of this effect is changes in psychological distress during follow-up resulting in the misclassification of exposure; that is, as time passes, there is an increasing opportunity for those who had psychological distress at baseline to no longer have distress, and vice versa.

We used a large cohort representative of the general population to examine the associations between psychological distress and cerebrovascular disease. However, because of the lack of follow-up data on psychological distress, we were unable to account for the effects of changes in distress over time. Furthermore, the GHQ- 12 is not designed to assess specific aspects of mental health such as anxiety and depression, although it has been specifically validated as a measure of depression as assessed by the Composite International Diagnostic Interview. ${ }^{17}$ Measuring symptoms of anxiety, depression and dysfunction as a unidimensional construct of psychological distress is particularly relevant in community-based samples such as ours, because mental health problems in the community are frequently characterized by shifting patterns of symptoms that resist precise clinical classification. ${ }^{27}$ We have previously shown that psychological distress (GHQ-12 score $>3$ ) was associated with incident cardiovascular events independent of the use of antidepressant medications (HR 1.48, 95\% CI $1.29-1.70){ }^{28}$ Thus, to some extent, the GHQ-12 might be independent of depression itself.

The participants excluded from our analyses were older, and thus probably had a greater risk of stroke and poorer mental health, which may have introduced bias into our analysis. In addition, we cannot discount the possibility of reverse causation - underlying disease at baseline might have increased psychological distress. However, we excluded all respondants with a clinical history of cardiovascular disease and performed our analyses again after removing all deaths that occurred in the first two years of follow-up; this additional analysis did not affect our results.

Finally, different subcategories of cerebrovascular disease could not be reliably separated using the information from death certificates (57.9\% of all deaths due to cerebrovascular disease were unspecified). Further work is required to determine whether psychological risk factors have a uniform affect on different subcategories of cerebrovascular disease.

\section{Conclusion}

Little is known about the psychological risk factors for cerebrovascular disease. In this large cohort representative of the general population, we saw an association between psychological distress and risk of death from cerebrovascular disease. Our data suggest that questionnaires such as the GHQ-12 could be of value in systematic screening aimed at improving the recognition of common mental disorders for reducing the risk of cardiovascular disease. Our results have several implications for further research. In particular, data from controlled trials that examine the effects of reducing distress on cardiovascular disease outcomes are needed to confirm the results we have seen.

\section{References}

1. Brotman DJ, Golden SH, Wittstein IS. The cardiovascular toll of stress. Lancet 2007;370:1089-100.

2. Everson SA, Roberts RE, Goldberg DE, et al. Depressive symptoms and increased risk of stroke mortality over a 29 -year period. Arch Intern Med 1998;158:1133-8.

3. Larson SL, Owens PL, Ford D, et al. Depressive disorder, dysthymia, and risk of stroke: thirteen-year follow-up from the Baltimore epidemiologic catchment area study. Stroke 2001;32:1979-83.

4. Ohira T, Iso H, Satoh S. Prospective study of depressive symptoms and risk of stroke among Japanese. Stroke 2001;32:903-8.

5. Simons LA, McCallum J, Friedlander Y, et al. Risk factors for ischemic stroke: Dubbo study of the elderly. Stroke 1998;29: 1341-6.

6. Bos MJ, Linden T, Koudstaal PJ. Depressive symptoms and risk of stroke: the Rotterdam study. J Neurol Neurosurg Psychiatry 2008;79:997-1001. 
7. Wouts L, Oude Voshaar RC, Bremmer MA, et al. Cardiac disease, depressive symptoms, and incident stroke in an elderly population. Arch Gen Psychiatry 2008;65:596-602.

8. Colantonio A, Kasi SV, Ostfeld AM. Depressive symptoms and other psychosocial factors as predictors of stroke in the elderly. Am J Epidemiol 1992;136:884-94.

9. Colantonio A, Kasl SV, Ostfeld AM, et al. Psychosocial predictors of stroke outcomes in an elderly population. J Gerontol 1993;48:S261-8.

10. Salaycik KJ, Kelly-Hayes M, Beiser A. Depressive symptoms and risk of stroke: the Framingham study. Stroke 2007;38:16-21.

11. Nabi H, Kivimäki M, Suominen S, et al. Does depression predict coronary heart disease and cerebrovascular disease equally well? The Health and Social Support Prospective Cohort Study. Int J Epidemiol 2010;39:1016-24.

12. Pan A, Sun Q, Okereke OI, et al. Depression and risk of stroke morbidity and mortality: a meta-analysis and systematic review. JAMA 2011;306:1241-9.

13. Surtees PG, Wainwright NW, Luben RN, et al. Psychological distress, major depressive disorder and risk of stroke. Neurology 2008;70:788-94.

14. Department of Health. Health survey for England 2001. London (UK): The Stationery Office; 2001.

15. Goldberg DP, Gater R, Sartorius N, et al. The validity of two versions of the GHQ in the WHO study of mental illness in general health care. Psychol Med 1997;27:191-7.

16. Hankins M. The factor structure of the twelve item General Health Questionnaire (GHQ-12): The result of negative phrasing? Clin Pract Epidemiol Ment Health 2008;4:10.

17. Aalto AM, Elovainio M, Kivimäki M, et al. The Beck Depression Inventory and General Health Questionnaire as measures of depression in the general population: a validation study using the Composite International Diagnostic Interview as the gold standard. Psychiatry Res 2012; Feb. 22. [Epub ahead of print].

18. May M, McCarron P, Stansfeld S, et al. Does psychological distress predict the risk of ischemic stroke and transient ischemic attack? The Caerphilly study. Stroke 2002;33:7-12.

19. Ferrie JE, Kivimäki M, Shipley MJ. Sleep and death. In: Cappucio FP, Miller M, Lockley S, editors. Sleep epidemiology from aetiology to public health. Oxford (UK): Oxford University Press; 2011. p. 50-73.

20. Mykletun A, Bjerkeset O, Dewey M, et al. Anxiety, depression, and cause-specific mortality: the HUNT study. Psychosom Med 2007;69:323-31.

21. Simons LA, Simons J, Friedlander Y, et al. A comparison of risk factors for coronary heart disease and ischaemic stroke: the Dubbo study of Australian elderly. Heart Lung Circ 2009;18:330-3.

22. Wilhelmsen L, Koster M, Harmsen P, et al. Differences between coronary disease and stroke in incidence, case fatality and risk factors, but few differences in risk factors for fatal and non-fatal events. Eur Heart J 2005;26:1916-22.

23. Everson SA, Lynch JW, Kaplan GA, et al. Stress-induced blood pressure reactivity and incident stroke in middle-aged men. Stroke 2001;32:1263-70.

24. Hamer M, Batty GD, Stamatakis E, et al. Hypertension awareness and psychological distress. Hypertension 2010;56:547-50.

25. Nabi H, Singh-Manoux A, Shipley M, et al. Do psychological factors affect inflammation and incident coronary heart disease? The Whitehall II study. Arterioscler Thromb Vasc Biol 2008;28: 1398-406.

26. Hamer M, Molloy GJ, Stamatakis E. Psychological distress as a risk factor for CVD events: pathophysiological and behavioral mechanisms. J Am Coll Cardiol 2008;52:2156-62.

27. Prince M, Patel V, Saxena S, et al. No health without mental health. Lancet 2007;370:859-77.

28. Hamer M, David Batty G, Seldenrijk A, et al. Antidepressant medication use and future risk of cardiovascular disease: the Scottish Health Survey. Eur Heart J 2011;32:437-42.

Affiliation: From the Department of Epidemiology and Public Health, University College London, London, UK

Contributors: Mark Hamer had full access to the data, and takes responsibility for the integrity of the data and accuracy of the analyses. All of the authors contributed to the concept and design of the study, drafted the article and revised it critically for intellectual content, and approved the final version submitted for publication.

Funding: The Health Survey for England is part of a program of surveys commissioned by The UK National Health Service Information Centre for Health and Social Care, and has been carried out since 1994 by the Joint Health Surveys Unit of the National Centre for Social Research and the Department of Epidemiology and Public Health at the University College London Medical School. Mark Hamer is supported by the British Heart Foundation (RE/10/005/28296); G. David Batty is a Wellcome Trust Career Development Fellow (WBS U.1300.00.006.00012.01); Emmanuel Stamatakis is a National Institutes of Health Research Career Development Fellow; Mika Kivimaki is supported by the UK Medical Research Council, the US National Institutes of Health (R01HL036310; R01AG034454), the New Occupational Safety and Health European Research Area program, and the Academy of Finland.

The funders played no role in the design and conduct of the study; collection, management, analysis and interpretation of the data; and preparation, review or approval of the manuscript. The views expressed in this article are those of the authors and not necessarily those of the funding bodies.

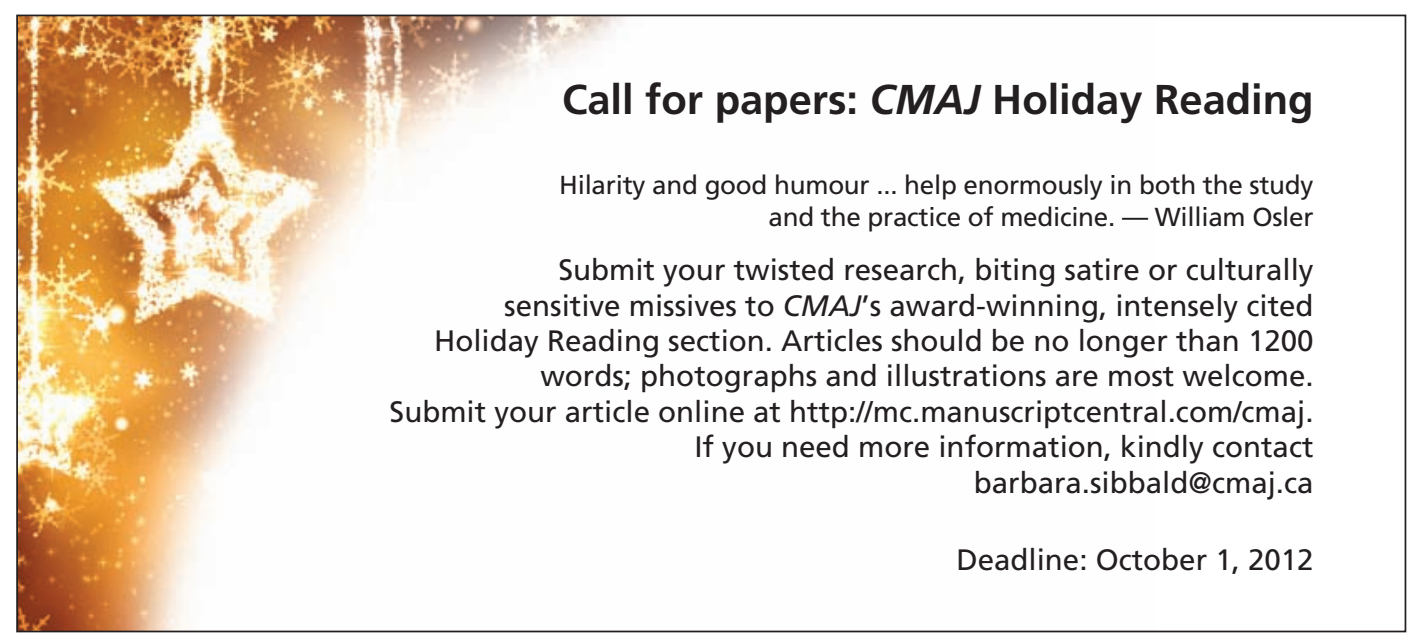

